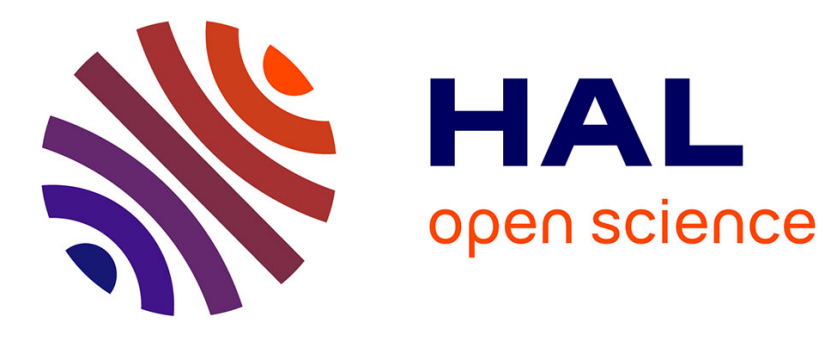

\title{
Local Density Estimation for VANETs
}

\author{
Noureddine Haouari, Samira Moussaoui, Mohamed Guerroumi, Sidi \\ Mohammed Senouci
}

\section{To cite this version:}

Noureddine Haouari, Samira Moussaoui, Mohamed Guerroumi, Sidi Mohammed Senouci. Local Density Estimation for VANETs. Global Information Infrastructure and Networking Symposium (GIIS), Oct 2016, Porto, Portugal. 10.1109/GIIS.2016.7814935 . hal-01543398

\section{HAL Id: hal-01543398 \\ https://u-bourgogne.hal.science/hal-01543398}

Submitted on 24 Feb 2018

HAL is a multi-disciplinary open access archive for the deposit and dissemination of scientific research documents, whether they are published or not. The documents may come from teaching and research institutions in France or abroad, or from public or private research centers.
L'archive ouverte pluridisciplinaire HAL, est destinée au dépôt et à la diffusion de documents scientifiques de niveau recherche, publiés ou non, émanant des établissements d'enseignement et de recherche français ou étrangers, des laboratoires publics ou privés.

\section{다)(1) $(5$}

Distributed under a Creative Commons Attribution - NonCommercial| 4.0 International 


\section{Local Density Estimation for VANETs}

\author{
Noureddine Haouari, Samira Moussaoui, Mohamed Guerroumi \\ RIIMA \\ Faculty of Electronics and Computer Science \\ University of Science and Technology Houari Boumediene USTHB, Algeria. \\ nhaouari@usthb.dz,smoussaoui@usthb.dz,mguerroumi@usthb.dz
}

\author{
Sidi Mohammed Senouci \\ DRIVE EA1859 \\ Univ. Bourgogne Franche Comt \\ 58000, Nevers, France. \\ Sidi-Mohammed.Senouci@u-bourgogne.fr
}

\begin{abstract}
Local vehicle density estimation is an integral part of various applications of Vehicular Ad-hoc Networks (VANETs) such as congestion control and congestion traffic estimation. Currently, many applications use beacons to estimate this density. However, many studies show that the reception rate of these beacons can significantly drop at short distances due to a broadcast storm problem in high-density situations. Therefore, the local vehicle density estimation helps VANETs' applications in giving an estimate of the number of neighbors in their communication range where a vehicle could send and receive correctly packets. Indeed, an accuracy local density estimation considerably enhances the performance of these applications and makes them adaptable to different road scenarios. Our aim in this work is to extend more the local density to be segmented and within the maximum transmission range. This potential gives VANETs' application the ability to estimate at different ranges depending on their requirements. To this goal, this paper proposes a segment-based approach that ensures high accuracy with low overhead over the maximum vehicles transmission range. Performance results show that the proposed strategy reaches a mean error ratio of approximately $3 \%$ with limited overhead over $1000 \mathrm{~m}$ of range.
\end{abstract}

\section{Introduction}

The advancement in wireless communication technologies made it possible to consider wireless networks providing connectivity among vehicles on the road. Such networks, often referred to as VANETs (Vehicular Ad-hoc Networks), represent an emerging and challenging technology that is helpful in developing intelligent transportation systems (ITS) with the aim of improving safety and comfort on our roads. One of the most challenging characteristics is related to the high-density variation since the density can go from very sparse (e.g. rural areas) to very dense (e.g. downtowns). How to accurately estimate the density by vehicles plays a primary role in many VANETs applications, such as road traffic assessment. Accurate estimation in these applications can help reduce travel time, fuel consumption, and pollution by allowing drivers to choose less congested roads. Additionally, estimation accuracy improves the functioning of all density-dependent applications/protocols such as congestion control protocols, routing, and dissemination protocols in order to efficiently perform (that is, improve the robustness, delay, and routing efficiency).

In many studies, local density estimation is defined as the estimated number of neighbors in the communication range based on exchanged beacons, used for mutual awareness, between vehicles. However, according to [1], in scenarios where there is a high message load, the reliable transmission range is reduced by up to $90 \%$. This degradation causes a severe lack of neighborhood awareness, which might affect the functioning of density-dependent VANETs applications. In fact, these applications require an accurate local density estimation strategy to perform efficiently. Due to the lack of using beacons, many density estimation approaches were proposed in the literature. The reviewed strategies can be divided into two categories: speed-based strategies [2], [3], [4] and communication-based strategies [5], [6], [7], [8], [9], [10], [11]. Speed-based mechanisms are based on the use of the speed/density relationship to estimate the density on the road. Communication-based strategies are based on exchanging messages between vehicles to estimate the density. Due to page limitation, We focus on the the strategy proposed in [10]. The authors propose DVDE strategy based on the segmentation of the transmission range into a number of segments and then the vehicles send the density of each segment every $n$ beacon, instead of sending neighbors D-FPAV [9]. By gathering this information from different vehicles, the vehicle can enhance the accuracy of its estimation. This approach reduces the overhead compared to the D-FPAV [9]. However, the process of data density sharing between vehicles is complex due to the different segment positions as it is supposed that each vehicle has its own segments. For that, linear interpolation is used to estimate the density of a target segment even if the segments are different, which happens in most cases. Also, this approach can give less accurate results if the vehicles are not uniformly distributed. Moreover, the shared information of periodic extended beacons can be useless if the vehicles are in the same area sharing the same information. This periodic redundancy creates an extra overhead that can be avoided if only selected vehicles share their information.

In this paper, we extend more the local density to be within the maximum transmission range and in segments 
manner to estimate at different distances. We propose a novel local density estimation approach within the maximum transmission range. We call it Segment-based Local Density Estimation (SLDE). The primary goal of SLDE is to provide an accurate local density estimation with the minimum overhead for density-dependent VANETs applications within the maximum radio transmission range of the vehicle (the maximum theoretical transmission range for IEEE 802.11p technology is $1000 m$ ). SLDE is based on the segmentation of the road with an efficient density propagation strategy. SLDE uses segments that are smaller than the maximum transmission range since this allows the vehicles to estimate accurately the density of the segment, which they are in. Also, this allows the vehicles to estimate density at different distances. The local density estimation will be calculated based on the exchange of density information of the segments in the maximum transmission range of the vehicle. SLDE shows advantages over existing strategies in two important aspects: (i) Use of fixed segments: the roads are considered as being segmented (Figure 1). This feature makes the share and use of the density information accessible and permits to estimate the density at different distances. (ii) Accurate estimation :Using of an efficient propagation strategy, the density propagation module is optimized to ensure that the density information is accurate, up to date, and uses the minimum overhead.

This paper is organized into six sections as follows: in Section 2, We describe the proposed SLDE strategy.Performance analysis and simulation results are presented in Section 3. Section 4 concludes the paper with outlooks on the future work.

\section{Segment-based Local Density Estimation (SLDE)}

SLDE is designed to enable VANETs applications to collect an accurate estimation of neighbors within the maximum transmission range with a minimum amount of overhead. This information is specifically provided to the density-dependent protocols and applications (e.g. traffic monitoring and congestion detection) to allow them to be adaptive to the ongoing traffic density state.

SLDE is designed under the following assumptions:

- Vehicles are equipped with omnidirectional antennas with the same receiving sensitivity and transmission range.

- Each vehicle knows its velocity and its geographical location through a Global Positioning System (GPS).,

- All vehicles are equipped with preloaded digital maps that provide the identity of each segment.

- The maximum transmission range is set to $1000 \mathrm{~m}$.

Safety applications require a reliable communication system to have strong knowledge of the local neighborhood. Vehicular state information (the position, speed, route, etc.) is broadcast periodically via messages called Cooperative
Awareness Messages (CAMs) in the USA, Basic Safety Messages (BSMs) messages in Europe, or generally, beacons. For our strategy, we sent additional information about the density via these messages; we call these messages extended beacons. In addition to beacons information, extended beacons have a density histogram $(D H)$, chosen reference vehicles $(R V s)$, and the number of reference vehicles $R V_{n} b$. The density histogram is an array that contains the densities of the segments within the maximum transmission range of a specific vehicle. The chosen $R V s$ are the candidate vehicles to be $R V$ in the next segment. $R V_{n} b$ is the number of the used reference vehicles in the construction of extended beacons.

The problem of packet loss affects beacons in long distances or even short distances when the control channel is congested. SLDE uses segments that are smaller than the maximum transmission range. This choice allows the vehicles to estimate accurately the density of the segment in which they are.

The basic idea of SLDE is to estimate the density based on the exchange of density information of the segments (Figure 1) in the maximum transmission range of the vehicle. The goal is to have a more accurate estimate of the density by using data from the nearest vehicle for each segment center. We develop the propagation of density information in SLDE to make it as synchronous as possible with a smaller amount of overhead.

SLDE's strategy is described in Algorithm 1. The density propagation begins with a $R V$ (Reference Vehicle). A vehicle might become $R V$ when it passes through the center of a segment or when it receives a $D H$ (Density Histogram), a vector that contains the densities of the segments in the maximum transmission range, from a different $R V$ in another segment. In the first case, the propagation of $D H$ begins, if the last received extended beacon about this segment is outdated. In the second case, the nearest vehicle to the center is chosen in each segment to propagate the density information with the goal of making the density information as synchronous as possible. Even if the density information is not yet outdated, the vehicle might send an extended beacon if it is more accurate than the last sent extended beacon. The accuracy of the extended beacon is measured by the number of used $R V s$ in its construction. Before sending a $D H$, the $R V$ fuses the different received $D H s$ by using the most accurate and available information through a data fusion process.

In the following, we describe the three main features of SLDE: fixed segments, data fusion and density propagation.

In SLDE, the roads are supposed to be segmented (Figure 1). Vehicles use preloaded digital maps to find the identity of each segment. The identification process of the segments is out of the scope of this paper. Using fixed segments unifies the segments for all vehicles, which allows for more accurate density information by avoiding using linear interpolation in the estimation.

Data fusion is done during $D H$ construction. The fusion of the received data is based on choosing the most accurate 


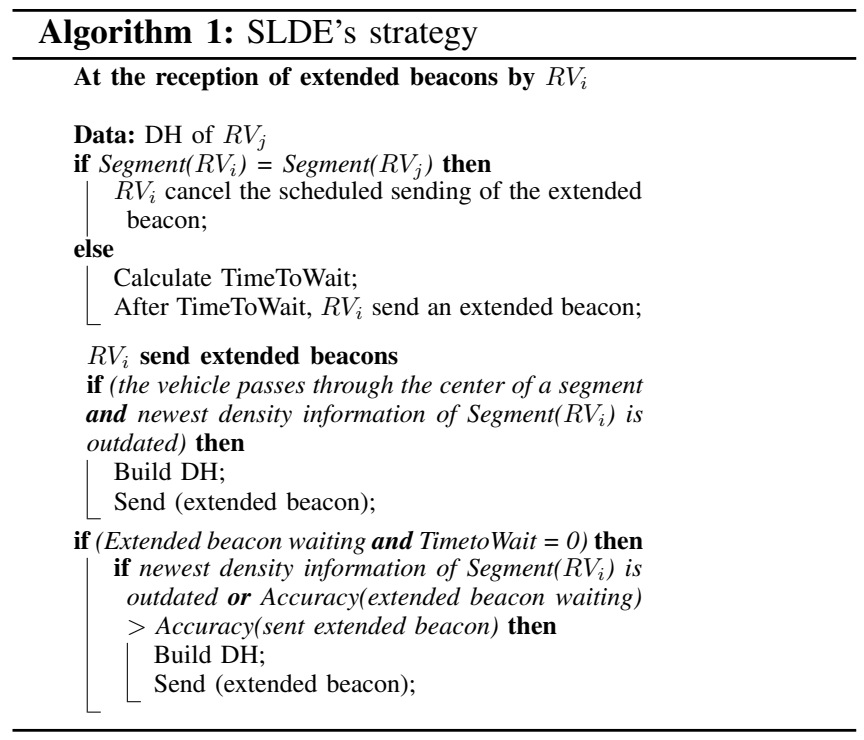

information for each segment. The estimator vehicle uses the valid data of the nearest $R V$ for each segment. The nearest $R V$ to the center has a higher probability of detecting the vehicles in that segment. The estimator vehicle will use the received regular beacons in the case of no reception of $D H$ that has better accuracy. By the end, the estimator vehicle will have a $D H$ that contains the densities of the different segments.

The density propagation is an important element to make the accurate density information available in a short time. In SLDE, there are two ways to start the propagation of density information. First, when a vehicle passes through the center of a segment; second, when a vehicle receives a $D H$.

The freshness of density information is critical to have accurate density. In SLDE, the nearest vehicle to the center is chosen to be the next $R V$. The choice of next $R V$ in SLDE is based on two methods. One method is based on timers, which requires that each vehicle receiving $D H$ sets a timer that corresponds to the distance to the center of the segment (TimeToWait). The other method is based on the sent $D H$, where the sending $R V$ includes in its $D H$ the next three $R V s\left(R V_{1}, R V_{2}, R V_{3}\right)$ in the next segment. The $R V s$ are sorted based on their distance to target segment center in order to make the delay time as short as possible. After the reception of $D H$, and once the timer expires, the corresponding vehicle responds by sending its $D H$ if the density information is still outdated. The density information is still valid when received within $\triangle T$ period. The timers of all other vehicles of the same segment are canceled, and their responses are suppressed. The equation 1 is used to calculate TimeToWait period:

$$
\text { TimeToWait }=\frac{D x, \text { segment }}{S L / 2} * L T E B
$$

Where:

Dx, segment: The distance between the vehicle and the center of its segment (m).

\section{$S L$ : Segment length (m).}

LTEB: Lifetime of an extended beacon(s).

Equation 1 is used to calculate the time to wait to ensure that the nearest vehicle to the center waits less. The smallest value is 0 when the vehicle is in the center $(D x$, segment $=$ $0)$. The greatest value is the lifetime of an extended beacon when the vehicle is on the border of a specific segment (Dx, segment $=\mathrm{SL} / 2$ ). In order to make the TimeToWait as short as possible and the extended beacons constructed segment by segment, the sending $R V$ includes in its $D H$ the next three $R V s\left(R V_{1}, R V_{2}, R V_{3}\right)$ in the next segment sorted based on their distance to its center. This information is to ensure that $D H$ is reconstructed in each segment. This permits to increase the accuracy by including the accurate information of the corresponding $R V s$. The TimeToWait calculation is changed to make the chosen $R V$ wait less, and the vehicles outside the target segment wait more (Algorithm 2 ). Additionally, if the sending $R V$ sent an extended beacon more accurate than the last sent extended beacon, it will still be sent even if the density information still valid, as shown in 1 . This is to propagate the accurate extended beacons as fast as possible to make their information available before becoming outdated.

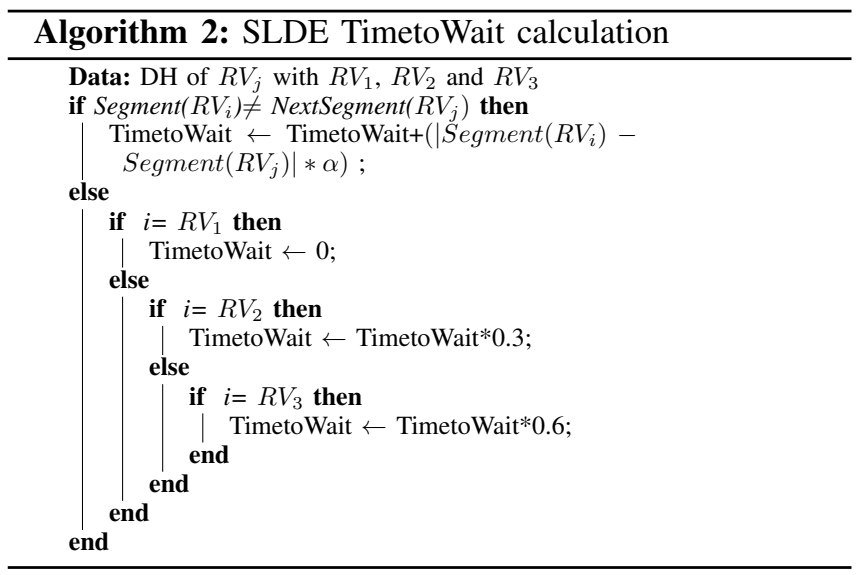

In the example illustrated in Figure 1, the density process begins with vehicle $\mathrm{A}$ (the first $R V$ ), which passes through the center of a segment. $R V A$ estimates the density based on the valid received $D H s$ via extended beacons and regular beacons. After that, it broadcasts the density information by including $R V 1$ as the first reference vehicle. The regular beacons are used for the estimation of the main segment and the segments where the estimator vehicle has better accuracy than the received $D H s$. When the vehicles receive the $D H$ in the next segment, they compete to be the next $R V$. In this example, vehicle $B$ receives the extended beacons, and elects itself as the next $R V$ by the broadcast of its $D H$ without waiting, thus suppressing other vehicles of the same segment. The other vehicles of the other segment will wait more time, which gives time for $D H$ to pass from one segment to another. Following the same strategy, the nearest vehicles to the center of each segment will react. 


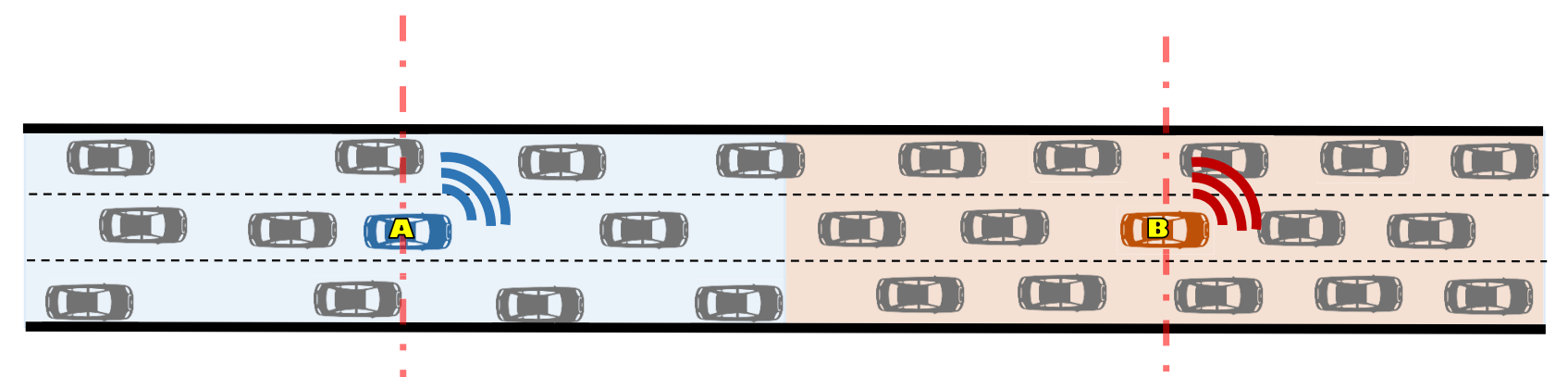

Figure 1: Illustration of a sample scenario of the SLDE process

\subsection{F-SLDE}

F-SLDE (Fundamental SLDE) is a variant of SLDE in which the propagation of extended beacons is based only on the timers. The sending $R V$ does not include the next $R V s$. This will make the selected $R V s$ of different segments send their $D H s$ directly without waiting for the reception of $D H s$ from the previous $R V s$ that are between the sending $R V$, and the receiving $R V$. In other words, there is no segment-by-segment propagation.

\section{Performance Evaluation}

In the following, we investigate the performance SLED compared to F-SLED and DVDE via simulation. We use the network simulator ns-2.35 [12], which is a well-known simulator among the network research community. We also use an overhauled MAC PHY-model [13] adapted to the characteristics of IEEE 802.11p (the standard of the intervehicle communications). During all the simulations, we used the probabilistic Nakagami propagation model [14] to ensure more realistic simulations. The simulated strategies are DVDE [10], F-SLDE, and SLDE. Throughout this section, we used the simulation configuration of DVDE [10] to ensure a fair comparison of performance. The used simulation parameters are listed in Table 1.

We consider the following metrics in our performance evaluation:

- Communication Overhead: It measures the generated overhead per second by all vehicles (bytes/second). This metric helps in the evaluation of the scalability issue. The overhead is computed as the mean number of sent $D H s$ per second multiplied by the size of extra bytes used for the density information. The size of $D H s$ is dependent on the number of segments (the density information of each segment requires 1 byte).

- Error Ratio: It is calculated using formula 2. It shows the percentage of undetected neighbors. This metric is used to evaluate the estimation accuracy.

$$
\text { Errorratio }=\frac{|E N-R N|}{R N} * 100 \%
$$

Where:

$E N$ : The estimated number of neighbors in the maximum transmission range.
$R N$ : The real number of neighbors in the maximum transmission range.

Our simulation mobility scenario comprises a bidirectional highway of $2 \mathrm{~km}$ in length with three lanes per driving direction. The highway is straight without entrances or exits. The used mobility scenarios show different levels of service on highways. For each level of service, we used the highest possible density based on the highway capacity manual [15]. In this manual, five different levels of service on highways based on the max road density. These are defined as following: A $(7 \mathrm{v} / \mathrm{l} / \mathrm{km}), \mathrm{B}(11 \mathrm{v} / \mathrm{l} / \mathrm{km}), \mathrm{C}(16$ $\mathrm{v} / \mathrm{l} / \mathrm{km}), \mathrm{D}(22 \mathrm{v} / \mathrm{l} / \mathrm{km})$, and $\mathrm{E}(25 \mathrm{v} / \mathrm{l} / \mathrm{km})$. We have used the traffic simulation package SUMO [16] to generate realistic traffic patterns for both the microscopic and macroscopic environments.

\begin{tabular}{ll}
\hline Parameter & Value \\
\hline Frequency & $5.9 \mathrm{GHz}$ \\
Data rate & $3 \mathrm{Mbps}$ \\
Carrier Sense Threshold & $-96 \mathrm{dBm}$ \\
Noise floor & $-99 \mathrm{dBm}$ \\
SINR for preamble capture & $4 \mathrm{~dB}$ \\
SINR for frame body capture & $10 \mathrm{~dB}$ \\
Slot time & $16 \mathrm{us}$ \\
SIFS time & $32 \mathrm{us}$ \\
Preamble length & $40 \mathrm{us}$ \\
PLCP header length & $8 \mathrm{us}$ \\
MAC & $802.11 \mathrm{P}$ \\
Beacon generation & $10 \mathrm{beacons} / \mathrm{s}$ \\
Packet size & $400 \mathrm{byte}$ \\
Maximum vehicle velocity & $30 \mathrm{~m} / \mathrm{s}$ \\
Transmission Range & $1000 \mathrm{~m}$ \\
Road length & $2 \mathrm{~km}$ \\
Radio propagation & Nakagami \\
Number of vehicles & $84,132,192,256,300$ \\
\hline
\end{tabular}

TABLE 1: Simulation configuration.

\subsection{Simulation Results}

We analyzed the different parameters values for DVDE, F-SLDE, and SLDE to choose the best configuration for each strategy and then evaluate their performances. The found values are the mean results of the different considered densities. The size of a segment and the validity time of the density information $\triangle T$ have a direct impact on the performance of F-SLDE and SLDE. For that, we analyzed different segment size values and $\triangle T$ to choose the most appropriate ones to ensure a low error ratio with the minimum of overhead. We did the same for the DVDE strategy but considered the segment size as it is the only used parameter in this strategy. 


\begin{tabular}{|cc|ccc|ccc|}
\hline Segment & $\Delta T(\mathbf{s})$ & \multicolumn{3}{|c|}{ Error ratio(\%) } & \multicolumn{3}{c|}{ Overhead(bytes/s) } \\
SLEc(m) & SLDE & F-SLDE & DVDE & SLDE & F-SLDE & DVDE \\
\hline 200.00 & 0.10 & 3.16 & 5.68 & 18.50 & 2710.08 & 1049.09 & 2138.40 \\
100.00 & 0.10 & 2.96 & 3.62 & 17.74 & 10558.83 & 3871.85 & 4082.40 \\
50.00 & 0.10 & 4.40 & 4.98 & 18.54 & 37560.92 & 14388.62 & 7970.40 \\
25.00 & 0.10 & 11.70 & 11.56 & 18.14 & 122459.04 & 47674.62 & 15746.40 \\
200.00 & 0.30 & 3.76 & 7.74 & 18.50 & 1431.88 & 371.04 & 2138.40 \\
100.00 & 0.30 & 3.12 & 4.64 & 17.74 & 5176.53 & 1327.71 & 4082.40 \\
50.00 & 0.30 & 4.64 & 5.72 & 18.54 & 18498.26 & 5025.66 & 7970.40 \\
25.00 & 0.30 & 11.74 & 11.08 & 18.14 & 62774.34 & 18186.85 & 15746.40 \\
200.00 & 0.50 & 4.96 & 8.94 & 18.50 & 721.17 & 231.70 & 2138.40 \\
100.00 & 0.50 & 3.60 & 5.20 & 17.74 & 2296.73 & 823.34 & 4082.40 \\
50.00 & 0.50 & 5.18 & 6.08 & 18.54 & 7458.32 & 3085.91 & 7970.40 \\
25.00 & 0.50 & 11.74 & 10.28 & 18.14 & 25361.60 & 11828.31 & 15746.40 \\
200.00 & 0.70 & 5.44 & 10.24 & 18.50 & 578.65 & 174.23 & 2138.40 \\
100.00 & 0.70 & 3.76 & 5.58 & 17.74 & 1793.09 & 603.38 & 4082.40 \\
50.00 & 0.70 & 5.34 & 6.12 & 18.54 & 5548.15 & 2240.47 & 7970.40 \\
25.00 & 0.70 & 11.56 & 9.56 & 18.14 & 18746.64 & 8970.44 & 15746.40 \\
200.00 & 0.90 & 6.26 & 12.66 & 18.50 & 477.82 & 137.25 & 2138.40 \\
100.00 & 0.90 & 3.96 & 6.02 & 17.74 & 1460.04 & 475.15 & 4082.40 \\
50.00 & 0.90 & 5.38 & 6.44 & 18.54 & 4369.46 & 1783.72 & 7970.40 \\
25.00 & 0.90 & 11.26 & 8.98 & 18.14 & 14643.28 & 7358.04 & 15746.40 \\
\hline
\end{tabular}

TABLE 2: Simulation results of F-SLDE, SLDE and DVDE.

Overall, the results presented in Table 2 show that FSLDE and SLDE outperform DVDE regarding error ratio and overhead. SLDE has the lowest mean error ratio of the three strategies: $2.96 \%$ with an overhead of 10558.83 bytes/second by using $100 \mathrm{~m}$ as segment size and 0.1 for $\triangle T$. For the overhead, it is always higher using small segments because of the need of including all their information. However, F-SLDE has the lowest overhead: 137.25 bytes/second by using $200 \mathrm{~m}$ as segment size and 0.9 for $\triangle T$ but with higher error ratio (12.66\%).

In order to select the best parameters of each strategy, we calculate its weight $(\omega)$ using the following equation:

$$
\omega(S, \triangle T)=\frac{\text { errorration }}{\text { Max (errorratio })}+\frac{\text { Overhead }}{\text { Max }(\text { overhead })}
$$

Equation 3 considers the error ratio and the overhead equally in the calculation of the weight. $M A X$ function gives the maximum obtained value using the same strategy.

The weight function for the illustrated results in Table 2 was calculated for all found results. For DVDE [10], the best results were found using segments of $200 \mathrm{~m}$. For FSLDE, $100 \mathrm{~m}$ as segment size and 0.1 as $\triangle T$ gives the lowest weight. For SLDE, the lowest weight was found using a segment size of $200 \mathrm{~m}$ with 0.1 as $\triangle T$. In the next section, the chosen best parameters will be used to evaluate the performance of the three strategies in more detail.

Figure 2 illustrates the generated overhead by varying the density of vehicles. We observe that the overhead increases with the density of vehicles. This is expected because increasing the number of vehicles increases the number of sent extended beacons in all strategies.

Figure 3, and Figure 4 show the evaluation of the error ratio for the selected parameters. Figure 3 shows a scatter plot of the real number of vehicles vs the estimated number of vehicles for each strategy. The plot shows clearly that SLDE and F-SLDE values are concentrated on the black line, which corresponds to the real values. However, DVDE strategy values are getting further from the line as the number of neighbors increases.

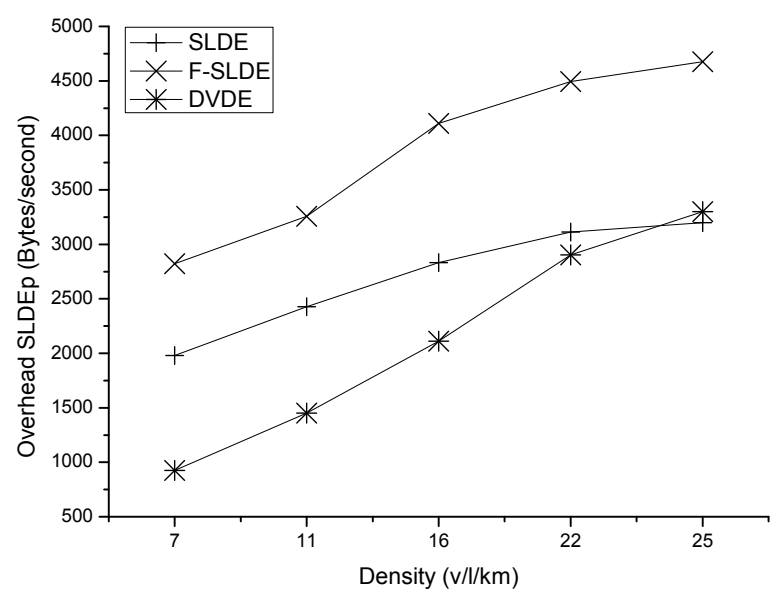

Figure 2: Overhead evaluation in different densities.

Figure 4 shows the error ratio of the strategies under the different densities. As it can be seen, SLDE has the lowest mean error ratio for most of the densities compared to the F-SLDE and DVDE strategies. The reduced error ratio for FSLDE and SLDE is mainly due to the propagation strategy, which ensures fresh, accurate density to different vehicles. SLDE gives better results than F-SLDE due to its capacity to use more $R V s$, which permits the $D H s$ to be constructed by more accurate information in each hop.

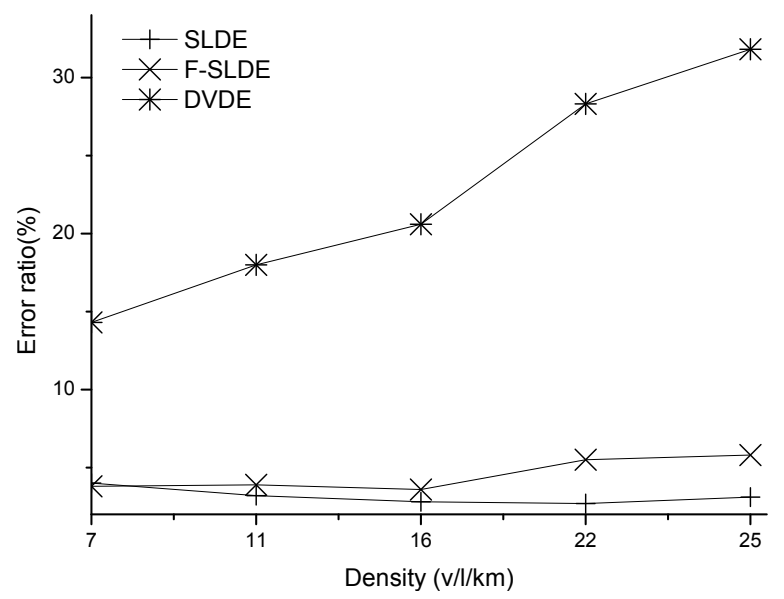

Figure 4: Accuracy evaluation in different densities.

In summary, F-SLDE and SLDE are characterized by a very low error ratio over the maximum transmission range with a low overhead. F-SLDE and SLDE take advantage of the different available information and make it more accurate by updating the $D H$ in each reference vehicle. SLDE gives the best results in terms of error ratio $(3.16 \%)$ with very low overhead ( 2710.08 bytes/second). This is the result of increasing the number of used $R V s$ by using segment-by-segment propagation strategy. 


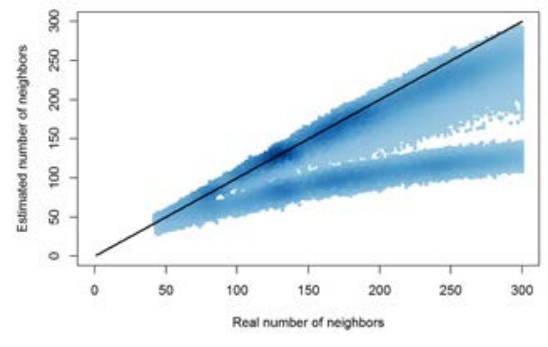

(a) DVDE

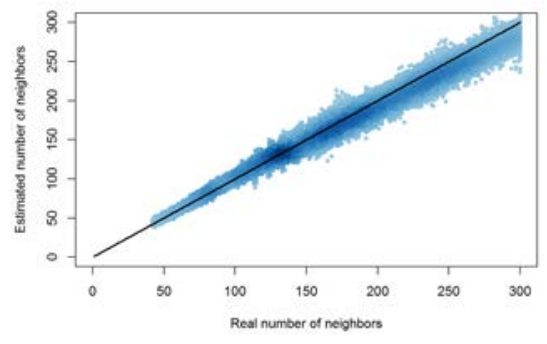

(b) F-SLDE

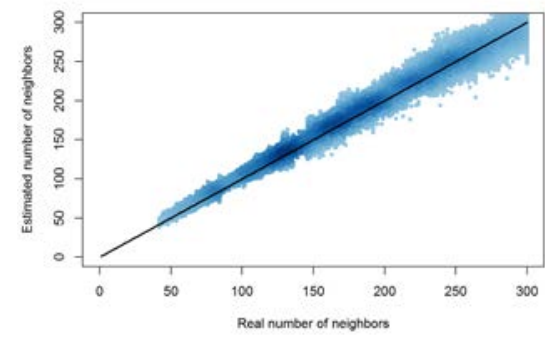

(c) SLDE

Figure 3: Comparison between the real number of neighbors and the estimated number of neighbors.

\section{Conclusion}

Local density estimation is crucial to ensure the efficiency of several applications in VANETs such as road traffic assessment, congestion protocols, routing and dissemination protocols. Their performance is directly related to the accuracy of this estimation. An efficient local density estimation strategy must permit high accuracy and limit the generated overhead.

We proposed a new local density estimation strategy called SLDE, a local density estimation strategy that efficiently estimates density over the maximum transmission range with very low overhead. SLDE introduces the use fixed segments and the propagation of density segment by segment. SLDE permits maximum accuracy while preserving very low generated overhead. A performance evaluation was done via simulation studies. The obtained simulation results demonstrate that SLDE can reach a mean error ratio of approximately $3.16 \%$ while ensuring a limited mean overhead of 2710.08 bytes/s. By using SLDE, the performance of many density-dependent applications and protocols will be increased. As a perspective for this work, we intend to use SLDE to enhance the performance of the congestion control in VANETs.

\section{References}

[1] R. K. Schmidt, B. Kloiber, F. Schuttler, and T. Strang, "Degradation of communication range in VANETs caused by interference 2.0 - realworld experiment," in Lecture Notes in Computer Science. Springer Science Business Media, 2011, pp. 176-188.

[2] M. Artimy, "Local density estimation and dynamic transmissionrange assignment in vehicular ad hoc networks," IEEE Transactions on Intelligent Transportation Systems, vol. 8, no. 3, pp. 400-412, Sept 2007.

[3] R. Shirani, F. Hendessi, and T. A. Gulliver, "Store-carry-forward message dissemination in vehicular ad-hoc networks with local density estimation," in Vehicular Technology Conference Fall (VTC 2009Fall), 2009 IEEE 70th, Sept 2009, pp. 1-6.

[4] T. Umer, Z. Ding, B. Honary, and H. Ahmad, "Implementation of microscopic parameters for density estimation of heterogeneous traffic flow for vanet," in Communication Systems Networks and Digital Signal Processing (CSNDSP), 2010 7th International Symposium on, July 2010, pp. 66-70.
[5] G. Khomami, P. Veeraraghava, and F. P. Fontan, "Node density estimation in VANETs using received signal power," Radioengineering, vol. 24, no. 2, pp. 489-498, jun 2015.

[6] R. Bauza and J. Gozalvez, "Traffic congestion detection in largescale scenarios using vehicle-to-vehicle communications," Journal of Network and Computer Applications, vol. 36, no. 5, pp. 1295-1307, sep 2013.

[7] S. Panichpapiboon and W. Pattara-atikom, "Evaluation of a neighborbased vehicle density estimation scheme," in ITS Telecommunications, 2008. ITST 2008. 8th International Conference on, Oct 2008, pp. 294-298.

[8] L. Garelli, C. Casetti, C. F. Chiasserini, and M. Fiore, "Mobsampling: V2v communications for traffic density estimation," in Vehicular Technology Conference (VTC Spring), 2011 IEEE 73rd, May 2011, pp. $1-5$.

[9] M. Torrent-Moreno, J. Mittag, P. Santi, and H. Hartenstein, "Vehicleto-vehicle communication: Fair transmit power control for safetycritical information," IEEE Transactions on Vehicular Technology, vol. 58, no. 7, pp. 3684-3703, Sept 2009.

[10] J. Mittag, F. Schmidt-Eisenlohr, M. Killat, J. Härri, and H. Hartenstein, "Analysis and design of effective and low-overhead transmission power control for vanets," in Proceedings of the Fifth ACM International Workshop on VehiculAr Inter-NETworking, ser. VANET '08. New York, NY, USA: ACM, 2008, pp. 39-48.

[11] M. Jerbi, S.-M. Senouci, T. Rasheed, and Y. Ghamri-Doudane, "An infrastructure-free traffic information system for vehicular networks," in 2007 IEEE 66th Vehicular Technology Conference. Institute of Electrical \& Electronics Engineers (IEEE), sep 2007.

[12] K. Fall and K. Varadhan, "The network simulator (ns-2)," URL: http://www.isi.edu/nsnam/ns, 2012.

[13] Q. Chen, F. Schmidt-Eisenlohr, D. Jiang, M. Torrent-Moreno, L. Delgrossi, and H. Hartenstein, "Overhaul of ieee 802.11 modeling and simulation in ns-2," in Proceedings of the 10th ACM Symposium on Modeling, Analysis, and Simulation of Wireless and Mobile Systems, ser. MSWiM '07. New York, NY, USA: ACM, 2007, pp. 159-168.

[14] M. NAKAGAMI, "The m-distributiona general formula of intensity distribution of rapid fading," in Statistical Methods in Radio Wave Propagation, W. HOFFMAN, Ed. Pergamon, 1960, pp. 3 - 36.

[15] H. C. Manual, "Highway capacity manual," 2000.

[16] D. Krajzewicz, J. Erdmann, M. Behrisch, and L. Bieker, "Recent development and applications of SUMO - Simulation of Urban MObility," vol. 5, no. 3\&4, December 2012, pp. 128-138. 